\title{
Characterization of two cytochrome P450 monooxygenase genes of the pyripyropene biosynthetic gene cluster from Penicillium coprobium
}

\author{
Jie $\mathrm{Hu}^{1,2}$, Hiroto Okawa ${ }^{2}$, Kentaro Yamamoto ${ }^{3}$, Kazuhiko Oyama $^{3}$, Masaaki Mitomi ${ }^{3}$ and Hiroyuki Anzai ${ }^{2}$ \\ Pyripyropenes are potent inhibitors of acyl-CoA:cholesterol acyltransferase, which were initially discovered to be produced by \\ Aspergillus fumigatus. Recently, Penicillium coprobium PF1169 has also found to produce pyripyropene A (PyA), which exhibits \\ insecticidal properties. Pyripyropenes are natural hybrid products of both terpenoid and polyketide origin. In our research, based \\ on data generated using the Genome Sequencer FLX for P. coprobium PF1169, we predicted the biosynthetic gene cluster of \\ PyA by blast analysis comparing with polyketide synthase and prenyltransferase of other species. By screening the genomic \\ fosmid library, nine open reading frames ( $p p b 1$ to $p p b 9$ ) related to the biosynthesis of PyA were deduced. Among them, two \\ cytochrome P450 monooxygenase genes ( $p p b 3$ and ppb4) were separately introduced into the model fungus $A$. oryzae. \\ Bioconversion of certain predicted intermediates in the transformants has elucidated the manner of hydroxylation in the \\ biosynthetic pathway by the expressed products of these two genes (P450-1 and P450-2). That is, P450-1 exhibits \\ monooxygenase activity and plays the hydroxylation role at $\mathrm{C}-11$ of pyripyropene $\mathrm{E}$. While $\mathrm{P} 450-2$ plays an active role \\ in the hydroxylation of $\mathrm{C}-7$ and $\mathrm{C}-13$ of pyripyropene 0. \\ The Journal of Antibiotics (2011) 64, 221-227; doi:10.1038/ja.2010.162; published online 12 January 2011
}

Keywords: cytochrome P450 monooxygenase; Penicillium coprobium; polyketide synthase; prenyltransferase; pyripyropene

\section{INTRODUCTION}

Pyripyropene A (PyA) has been found to be produced by Penicillium coprobium $\mathrm{PF} 1169^{1}$ and exhibits insecticidal properties. ${ }^{2}$ The isomers of pyripyropenes A to $\mathrm{L}$, initially isolated from the fermentation broth of Aspergillus fumigatus FO-1289 in 1993 (see ref. 3), have been regarded as potent inhibitors of acyl-CoA:cholesterol acyltransferase, the enzyme responsible for intracellular esterification of cholesterol. It has been reported that PyA displays insecticidal activity against Helicoverpa zea larvae. ${ }^{2}$ The isomers of pyripyropenes A, B and D from a marine-derived fungus Aspergillus sp. GF5 were recently found to exhibit selective anti-growth properties against human umbilical vein endothelial cells. ${ }^{4}$

Previous biochemical experiments partially delineated the PyA biosynthetic pathway. ${ }^{5}$ Recently, the early steps of its biosynthesis in A. fumigatus FO-1289 were precisely elucidated by applying a transgenic approach with heterologous fungus. Nevertheless, hydroxylation and acetylation mechanisms for the late steps had remained unknown.

We focused on cytochrome P450s, which are candidate catalysts at this hydroxylation step. Cytochrome P450 enzymes constitute a superfamily that typically catalyze the oxidation of numerous endogenous and exogenous compounds in bacteria, fungi, plants, insects and vertebrates. ${ }^{6-8}$ The cytochrome acts as a terminal oxidase accepting electrons from NADPH, through NADPH cytochrome P450 reductase, or from NADH through cytochrome $b_{5}$. The exogenous compounds metabolized in this way include numerous pesticides and insecticides. $^{9}$

In this paper, we describe the cloning and structural analysis of a $24 \mathrm{~kb}$ genomic DNA region of $P$. coprobium, which includes nine candidate genes for PyA biosynthesis. Among these nine genes, two cytochrome $\mathrm{P} 450$ monooxygenases were introduced into the model fungus $A$. oryzae and analyzed hydroxylation activity by feeding predicted intermediates of PyA to the culture of each tested transformant.

\section{MATERIALS AND METHODS}

Strains and growth conditions

P. coprobium PF1169 (Meiji Seika Kaisha, Ltd.) and A. oryzae sC (adenosine triphosphate sulfurylase) mutant strain HL-1105 (Hakutsuru Sake Brewing, Kobe, Japan) were used in this study. P. coprobium was grown on culture plates containing CMMY medium (corn meal 0.85\%, Bacto Agar 0.75\%, malt extract $1 \%$ and yeast extract $0.2 \%$ ) for 5 days at $28^{\circ} \mathrm{C}$. The P. coprobium was then inoculated into NB liquid media (nutrient broth $0.8 \%$, yeast extract $0.2 \%$ and $\mathrm{KNO}_{3} 0.05 \%$ ) in a $500 \mathrm{ml}$ Erlenmeyer flask, and cultured at $28^{\circ} \mathrm{C}$ for 3 days on a rotary shaker (120 r.p.m.). The mycelia were harvested and prepared for DNA extraction.

${ }^{1}$ Department of Applied Life Science, United Graduate School of Agricultural Science, Tokyo University of Agriculture and Technology, Tokyo, Japan; ${ }^{2}$ Gene Research Center, Ibaraki University, Ibaraki, Japan and ${ }^{3}$ Meiji Seika Kaisha, Ltd., Kanagawa, Japan

Correspondence: Professor H Anzai, Gene Research Center, Ibaraki University, 3-21-1 chuo, Ami, Inashiki, 300-0393 Ibaraki, Japan.

E-mail: anzai@mx.ibaraki.ac.jp

Received 10 July 2010; revised 9 November 2010; accepted 24 November 2010; published online 12 January 2011 
For bioconversion analysis, A. oryzae HL-1105 for transformation was grown in Czapek-Dox medium $\left(\mathrm{NaNO}_{2} 0.3 \%\right.$, KCL $0.2 \%, \mathrm{KH}_{2} \mathrm{PO}_{4} 0.1 \%$, $\mathrm{MgSO}_{4} \bullet 7 \mathrm{H}_{2} \mathrm{O} \quad 0.05 \%$, trace elements solution $1 \mathrm{mll}^{-1}$, glucose $2 \%$ and methionine $40 \mu \mathrm{g} \mathrm{ml}^{-1}$ ). As a negative control, wild-type A. oryzae HL-1034 was grown in Czapek-Dox medium without methionine. The fungal spores were cultured in YDP (yeast extract $0.5 \%$, dextrin $2 \%$ and polypepton $1 \%$ ) with $1 \%$ maltose.

\section{Isolation of genomic DNA from $P$. coprobium}

Genomic DNA was isolated using the QIAGEN Genomic DNA Kit (QIAGEN K. K, Tokyo, Japan). Mycelia, which were 3-4-days old were harvested from liquid cultures, and then transferred to a pre-cooled mortar for freezing in liquid nitrogen. The frozen mycelia were ground to a powder and resuspended in Buffer G2. All procedures were performed according to the manufacturer's instructions. The isolated DNA was checked for integrity on $0.8 \%$ agarose gel and the concentrations were determined spectrophotometrically.

\section{Construction of fosmid genomic library}

To generate a genomic library, chromosomal DNA of P. coprobium was randomly sheared by passing it through a $200 \mu \mathrm{l}$ small bore pipette tip. The sheared genomic DNA was examined on $0.5 \%$ agarose gel using the Fosmid Control DNA $(40 \mathrm{~kb})$ as a control and migrated with the control DNA. The sheared DNA was cloned into pCC1FOS according to the manufacturer's directions for the CopyControl Fosmid Library Production Kit (Epicentre Biotechnologies, Madison, WI, USA). To construct the complete library covering the entire $P$. coprobium genome, the $3 \times 10^{4} \mathrm{cfu} \mathrm{ml}^{-1}$ of package particles was infected into TransforMax EPI300-T1 E. coli cells (Epicentre Biotechnologies) and separately transferred into a 96-well deep plate to generate $\sim 100$ clones per well.

\section{Screening of the genomic library for a pyripyropene A biosynthetic gene cluster positive clone}

To identify fosmid clones carrying the pyripyropene A biosynthetic gene cluster, the fosmid DNA mixtures with about 100 independent clones per well were extracted and PCR was performed to screen the positive well. The infected cells of positive mixture well were plated on LB medium (yeast extract $0.5 \%, \mathrm{NaCl}$ $1 \%$, tryptone $1 \%$ and agar $2 \%$ ) supplemented with $12.5 \mu \mathrm{g} \mathrm{ml}^{-1}$ chloramphenicol and incubated at $37^{\circ} \mathrm{C}$ for $17-18 \mathrm{~h}$. The generated clones were transferred to Amersham Hybond-N+ nylon membrane (GE Healthcare, Little Chalfont, Buckinghamshire, UK) and hybridized to a labeled gene probe upstream of the UbiA-like prenyltransferase (PT) gene. This probe was labeled by using the AlkPhos Direct kit (GE Healthcare). Positive signals on the membrane were assigned to the respective clone on the agar plates. The positive clones were confirmed by colonial PCR with the primer pair designed for the probe. The positive clones were amplified and the DNA was isolated by standard techniques.

\section{Sequencing of positive clone of fosmid library}

Positive clones were sequenced with primers on the basis of data from the Roche Genome Sequencer FLX454 system (Roche, Branford, CT, USA). All sequence reactions were carried out using the ABIPRISM BigDye Terminator Cycle Sequencing Kit (Applied Biosystems, Foster City, CA, USA) and ABI 3130 Genetic Analyzer (Applied Biosystems). The overlapping sequences were aligned to obtain the full-length sequence of the fosmid clones. Further sequence analysis was performed using the ATGC network software package (version 4; Genetyx Corporation, Tokyo, Japan).

\section{Construction of expression plasmids}

For the expression of P450-1 and P450-2 in A. oryzae, the expression vector pUSA $^{10}$ with amylase promoter was used. The expression plasmids were constructed as follows. For expression of P450-1, the coding sequence of ppb3 gene was amplified with PCR using the primer pairs listed in Table 1 and the PCR product was inserted into pCR-Blunt vector (Invitrogen, Carlsbad, CA, USA). The resultant plasmid was digested with KpnI and SwaI, and inserted into KpnI- and SmaI-treated pUSA to give pUSA-P450-1. For expression of $\mathrm{P} 450-2$, the coding sequence of $p p b 4$ gene was amplified with
Table 1 List of primer pairs used in this experiment

\begin{tabular}{lll}
\hline Primer & Sequence $5^{\prime}-3^{\prime}$ & Used for \\
\hline $27 \mathrm{~F}$ & CCCAGCCCAAGACTTGAGTA & Screening library \\
$27 \mathrm{R}$ & CCATCCATGTTGTGAGGACA &
\end{tabular}

P450-1 with Kpn F GCGCGGTACCATTGAGACAACATGGAT P450-1 with Swa R ATTTAAATAGTTAGACAATAGTATCA

Full-length genomic DNA of P450-1

\section{$22 \mathrm{~F}$}

$\mathrm{P} 450-1$ in R2 GCGTTAGTCAAACGGCTCTC TGTCGCAACTTATGGAATCG

\section{P450-2 F}

P450-2 R

F5 for P450-2 R5 for P450-2

\section{TTGTCTTAGCATTTTCATTA TCGTAATTTTCCCTAGATCA}

TACTTGGACGGACTCACCAGACTGGCA TGAGGTAGCACTG AGTAAAGCGCGAAATGCTCCGGTTG TGACTGGATGCAAC
Confirmation of transformant P450-1

Full-length genomic DNA of P450-2

Confirmation of transformant P450-2
PCR using the primer pairs listed in Table 1 and the PCR product was directly inserted into pUSA, and named pUSA-P450-2. The resulting expression plasmids were introduced into $A$. oryzae HL-1105 $s C$ mutant, and the transformants were selected using Czapek-Dox medium.

\section{Transformation of A. oryzae}

A. oryzae protoplasts were obtained and transformed according to the standard method. ${ }^{11}$ Protoplasts from stain HL-1105 were transformed separately with the plasmids carrying the two respective P450 genes. Each independent transformant was grown, harvested and analyzed. The DNA for each transformants was extracted in accordance with the modified protocol. ${ }^{12}$ Transformants carrying the respective P450 genes were verified by PCR analysis using the MightyAmp DNA polymerase (Takara Bio, Ootsu, Shiga, Japan) using the primers listed in Table 1.

\section{Metabolite analysis}

To determine each open reading frames (ORFs) function, wild-type A. oryzae HL-1034 and positive transformants of A. oryzae HL-1105 with plasmids containing P450 genes were grown at $30^{\circ} \mathrm{C}$ on Czapek-Dox medium for 7 days. Fungal spores for each sample were transferred into a $50 \mathrm{ml}$ flask of $10 \mathrm{ml} \mathrm{YDP}$ containing $1 \%$ maltose and intermediates, and then cultured at $25^{\circ} \mathrm{C}$ while being agitated. After 24-96 h, the bioconversion products of the intermediates were processed using culture extracts from mycelia and broth of each spot (hereafter refers to as 'extract'). A volume of $1 \mathrm{ml}$ of acetone was added to $1 \mathrm{ml}$ of extract and thoroughly mixed. Acetone was removed by centrifugal concentrator and $1 \mathrm{ml}$ of ethyl acetate was added and mixed again. The extract ( $500 \mu \mathrm{l}$ of ethyl acetate layer) was then concentrated to dryness. Individual samples were dissolved in $1 \mathrm{ml}$ of methanol and analyzed with LC-MS on a Micromass ZQ (Waters, Milford, MA, USA), 2996 Photodiode array (Waters), 2695 Separation module (Waters) and a Xterra C18 column $(\phi 4.6 \mathrm{~mm} \times 50 \mathrm{~mm}$, $5 \mu \mathrm{m}$; Waters). For LC-NMR, the filtrate was dissolved in $\mathrm{CD}_{3} \mathrm{CN}+\mathrm{D}_{2} \mathrm{O}$ and the ${ }^{1} \mathrm{H}$ spectrum was measured at $500 \mathrm{MHz}$ with a Bruker Avance 500 (Bruker Biospin, Rheinstetten, Germany). Samples for LC-NMR were subjected to Agilent1100 separation module (Agilent Technologies, Palo Alto, CA, USA) equipped with Atlantis T3 column $(4.6 \times 150 \mathrm{~mm}, 5 \mu \mathrm{m}$; Waters).

\section{RESULTS}

\section{BLAST analysis of data obtained through Roche 454 FLX} sequencing

Total reads from 2.5 run were obtained through 454 FLX sequencing. The 13629 contigs were obtained, and the total length of the base pairs was about $30 \mathrm{Mbp}$. On the basis of the data from this large scale 


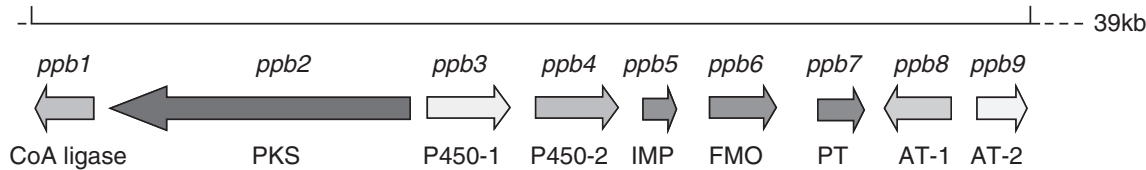

Figure 1 Gene cluster involved in pyripyropene A biosynthesis in P. coprobium PF1169. PKS, polyketide synthase; P450, cytochrome P450 monooxygenase; IMP, integral membrane protein; FMO, FAD-dependent monooxygenase; PT, UbiA-like prenyltransferase; AT, acetyltransferase. Arrows indicate the direction from the start to the stop codon. The number shows the location in the fosmid clone G7-9 in this study (GenBank accession no. FW308713).

sequence of $P$. coprobium, the tblastn search (protein $\rightarrow$ DNA) (Basic Local Alignment Search Tool, NCBI BLAST) was carried out comparing with five proteins known to be involved in polyketide and terpenoid biosynthesis. ${ }^{13,14}$ These five proteins were three polyketide synthases (RAM2-like protein of P. marneffei (GenBank accession no. Q0MR08)), the polyketide synthetase (PKS) PksP of A. fumigatus A1163 (GenBank accession no. Q4WZA8), 6-MSAS (6-methylsalicylic acid synthase) ${ }^{15}$ of P. patulum (GenBank accession no. P22367) and two PTs (UbiA PT family protein (GenBank accession no. XP_746965)) and the UbiA-like PT (GenBank accession no. XP_751272) of A. fumigatus Af293. Of these contigs, the scaffold 00057 (size $188 \mathrm{~kb}$ ) shows a high degree of homology to the proteins of PKSs and PT. The results of blastn research (NCBI BLAST) showed that PksP and 6-MSAS share the highest homology, with the $5^{\prime}$ region beginning at $117 \mathrm{~kb}$ of scaffold 00057 . The UbiA PT family protein and UbiA-like PT showed the highest homology with the distal $3^{\prime}$ end of this scaffold, starting at $124 \mathrm{~kb}$.

Isolation of fosmid clones covering PyA biosynthetic gene cluster To isolate genomic DNA regions carrying genes of the PyA biosynthetic pathway, the fosmid genomic library for P. coprobium was constructed. The fosmid DNA mixtures of each well were isolated and PCR was performed to screen the positive wells. We designed the screening primer pair $(27 \mathrm{~F} / 27 \mathrm{R}$, in Table 1$)$ near the UbiA-like PT gene. ${ }^{16}$ The positive mixture wells were then distributed to a LB solid medium containing chloramphenicol. The clones were screened by colony hybridization with the labeled probe. The fosmid DNA of the 6 positive clones were sequenced starting from the $5^{\prime}$ region and distal $3^{\prime}$ end using the sequencing primer of the fosmid vector. The data were compared with the total genomic DNA of $P$. coprobium through Roche Genome Sequencer FLX 454 system. Among these six clones, a clone G7-9 containing about $39 \mathrm{~kb}$ fragment was used for further analysis.

\section{Map of the putative gene cluster of PyA biosynthesis in $P$. coprobium}

The complete $39 \mathrm{~kb}$ sequence of inserted DNA of the G7-9 clone screened above was determined. The entire sequence (GenBank accession no. FW308713) was analyzed using the NCBI BLAST, enabling a search for ORFs. ${ }^{17,18}$ It has been reported that the putative biosynthetic gene cluster of PyA is composed of nine ORFs based on A. fumigatus F37 genomic database. ${ }^{19-21}$ In $P$. coprobium, among the $24 \mathrm{~kb}$ region of the inserted $39 \mathrm{~kb}$ (from 3301 to $27200 \mathrm{bp}$ in $39 \mathrm{~kb}$ region), nine ORFs were also identified. All of these ORFs (Figure 1) showed high homology to polyketide biosynthetic enzymes and other enzymes involved in terpenoid synthesis. These include CoA ligase ( $p p b 1)$, PKS (ppb2), P450-1 (cytochrome P450 monooxygenase 1) (ppb3), P450-2 $(p p b 4)$, integral membrane protein ( $p p b 5), \mathrm{FAD}$-dependent monooxygenase ( $p p b 6)$, PT ( $p p b 7)$, acetyltransferase-1 (AT-1) ( $p p b 8)$ and AT-2 $(p p b 9)$ in that order. In our research, we found the orientation of $p p b 8$ is to the left, rather than to the right as previously reported. ${ }^{21}$
In this paper, we focused on two genes encoding the P450s. On the basis of the deduced amino acid, the molecular weight of P450-1 (509 amino acids) was calculated to be $\sim 57.3 \mathrm{kD}$ and that of P450-2 (505 amino acids) was $\sim 56.6 \mathrm{kD}$. Analyzed by multiple alignment with amino acid sequences, P450-1 showed 95\% homology to cytochrome P450 monooxygenase of $A$. fumigatus Af293, whereas P450-2 showed $74 \%$ homology to A. fumigatus Af293 along the full length (Figure 2a). The similarity between these two P450s enzymes was 77\% (Figure 2b).

\section{Introduction of $p p b 3$ (P450-1) and $p p b 4$ (P450-2) into model fungus $A$. oryzae}

To clarify the functions of $p p b 3$ (P450-1) and $p p b 4$ (P450-2) in PyA biosynthesis from $P$. coprobium, these two genes were introduced into the model fungus $A$. oryzae. The PCR products of the $p p b 3$ and $p p b 4$ from fosmid G7-9 were inserted between the $\alpha$-amylase promoter and terminator in the fungal expression vector pUSA. The resulting plasmids were named pUSA-P450-1 and pUSA-P450-2 (Figure 3). Two $\mathrm{P} 450$ genes were transformed into A. oryzae separately by random recombination, after which the existence of each gene was confirmed by genomic PCR of the transformants (Figure 4). We also found that transformation efficiency was an average of 10 clones with $10 \mu \mathrm{g}$ DNA through the A. oryzae protoplast transformation system.

Functional analysis of P450-1 and P450-2 in model fungus $A$. oryzae After feeding intermediates with each transformant, the bioconversion of the intermediate was detected. LC-MS and LC-NMR analysis of the extracts of transformant $\mathrm{P} 450-1$, which was fed with pyripyropene $\mathrm{E}$ (PyE) and incubated for $48 \mathrm{~h}$, indicated the appearance of a compound earlier than that of PyE (Figure 5a). After $96 \mathrm{~h}$ of incubation, the first peak of compound increased, whereas the second peak of PyE declined (Figure $5 \mathrm{~b}$ ). In the ESI-MS spectrum, the $[\mathrm{M}+\mathrm{H}]^{+}$peak was observed at $\mathrm{m} / \mathrm{z}$ 468. The molecular formula was determined to be $\mathrm{C}_{27} \mathrm{H}_{33} \mathrm{NO}_{6}$. Its molecular weight is 16 greater than PyE. In the ${ }^{1} \mathrm{H}$ NMR spectrum, four methyl signals appeared in PyE (see Supplementary SI 1), whereas in the extracts from the transformants P450-1 three methyl signal and two proton signals (3.134 and 3.157 p.p.m. for H-11) were observed (see Supplementary SI 2). As is widely known, the structural differences between PyE and PyA lie at C-7, C-11 and C13. In BLAST research, $\mathrm{P} 450-1$ and $\mathrm{P} 450-2$ showed high homology with cytochrome P450 monooxygenses. We deduced P450-1 would activated at C-7, C-11, or C-13. One methyl group differing between PyE and PyA appears at C-11. On these basis, we deduced that one oxy-methine is located at $\mathrm{C}-11$ and is a deacetylated form of pyripyropene $\mathrm{O}(\mathrm{PyO})$. This was named 11-deacetyl-PyO (11-DeAc-PyO) (compound 9 shown in Figure 6). The 11-DeAc-PyO was not found in the extracts of the control transformant with the vector alone all the time. For the transformants P450-2, the PyO was fed to the culture. With the 48-h cultured extracts, the LC graph showed the emergence of a compound earlier than that of PyO (Figure 5d). The results of LC after 72 and $96 \mathrm{~h}$ incubation also showed that the peak of the new 


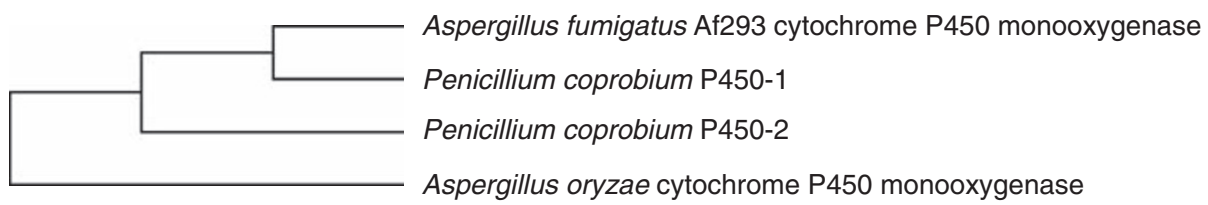

\section{b}

\section{GENETYX: Homology}

Penicillium coprobium P450-1 Penicillium coprobium P450-2 Penicillium coprobium P450-1 Penicillium coprobium P450-2 Penicillium coprobium P450-1 Penicillium coprobium P450-2 Penicillium coprobium P450-1 Penicillium coprobium P450-2 Penicillium coprobium P450-1 Penicillium coprobium P450-2 Sb Penicillium coprobium P450-1 Penicillium coprobium P450-2 Penicillium coprobium P450-1 Penicillium coprobium P450-2 St Penicillium coprobium P450-1 Penicillium coprobium P450-2 St Penicillium coprobium P450-1 Penicillium coprobium P450-2
Identity: $176 / 487$ (36\%)

Similarity: $376 / 487$ (77\%)

Query 20 VIISFLGLLTFSFVSPHLFPSRLTVINGGRAWDIFRTKAKKRFRSDAARLIKNGFEESPD 79

$\ldots+|\ldots| \ldots|\ldots| \ldots|\ldots| \ldots|.| \ldots|\ldots||\ldots| \ldots|\ldots| \ldots||$

Sbjet 18 LVLAGLYLIRLTLSSDQL--DKFPSINPRKPWEIVNVFAQRRFQQDGPRYLEAGYAKSP-

Query 80 AFRIITDNGPLLVLSPQYAREVRSDDRLSLDHFIASEFHPNIPGFEPFKLILDPKNPLNT $|\ldots||||||.| \ldots|\ldots| \ldots|\ldots| \ldots|+\ldots||\ldots| \ldots$ Sbjet 75 IFSVVTDLGPKLVVSGAFIEEFKDEKLLDHYRSMIEDFMAEVPGFESMFLGNLHNTVLRD Query 140 IL---KSNLTQALEDLSAEVTEALSATCTDDPEWHEVSVSQTALKIIAQMASKAFIGQER

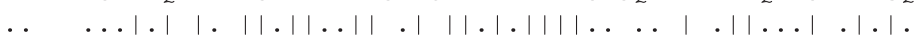
Sbjet 135 VISVITRELEQLLAPLSDEVSAALVDTWTDSPDWHEVALLPSMLGLIAKVSSLVFVGEPL 194 Query 197 CRDAKWHNIIITYTHNVYGAAQALHFWPSFLRPIVAQFLPACRTLQAQIAEAREILEPLV

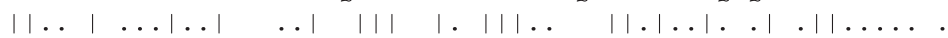
Sbjet 195 CRHPVWLETVINFTLIRHNAILALHQCPAVLRPVLHWVLPPCQKLRREIRTARTLIDSAL Query 257 AQRRAERATRAAQEKPHPSGGDI IDWLEQFYGDQPYDPVAAQLLLSFAAIHGTSNLLAQA

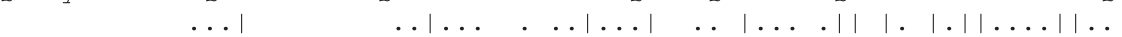
Sbjet 255 EKSR--------KNPQTEKESSVAWVDAFAKGNKYNAAMVQLRLANASIHSSADLLVKI 305 Query 317 LIDLCGQPELVQDLREEAVSVLGKEGWTRAALYQLKLMDSALKESQRLAPNRLLSMGRIA ||$+||||.|\ldots||| .||||.|\ldots||\ldots||||| .|| .|||||||| \quad \ldots \mid \ldots$ Sbjet 306 LINLCEQPELIRDLRDEIISVLGENGWRSSTLNQLKLLDSVLKESQRLHPVTTGAFSRFT Query 377 QGDMDLSDGLRIHRGTTLMVSAHNMWDPEIYPDPRKYDGYRFHKLRQTSGQEGQHQLVSS . $|\ldots| \ldots|\quad| \ldots|| \ldots|| \ldots \quad|\ldots|||||||,|\ldots| \ldots \ldots$ Sbjet 366 RQDIKLTNGTEIPSGTPIMVTNDVAGDASIYDDPDVFDGYRYFRMRE-GADKARAPFTTT Query 437 TPDHMGFGYGKHACPGRFFAAAQIKVALCNILLKYDIEYRGGKSPGVWGQGIHLFPDPTS

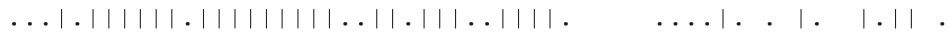
Sbjet 425 GQNHLGFGYGKYACPGRFFAATEIKIALCHMLLKYEWRLVKDRPHGIVTSGFAAFRDPRA 484 Query 497 RIHVRRR 503 . $\mid$. | | | | Sbjet 485 SIEVRRR 491

Figure 2 (a) TreePlot showing the homology of P450-1 and P450-2 in $P$. coprobium compared with cytochrome P450 monooxygenases from A. fumigatus Af293 (GenBank accession no. XP_001481476) and A. oryzae (GenBank accession no. BAJ04445) (UPGMA). (b) Amino acid sequence alignment of P450-1 and P450-2 in P. coprobium.

compound was at its highest point, whereas the peak for PyO declined (data not shown). With the 36-h cultured extracts, a small peak emerged earlier than the peak for PyO (Figure 5c). In the ESI-MS spectrum, the $[\mathrm{M}+\mathrm{H}]^{+}$peak was observed at $m / z 542$. The molecular formula was determined to be $\mathrm{C}_{29} \mathrm{H}_{35} \mathrm{NO}_{9}$. The molecular weight is 32 greater than that of $\mathrm{PyO}$. The ${ }^{1} \mathrm{H}$ NMR spectrum showed a proton signal at 3.65 p.p.m. (H-7). One additional proton signal was observed at 4.858 p.p.m. (H-13) (Supplementary SI 3 and SI 4). The structural difference between $\mathrm{PyO}$ and PyA lies at C-7 and C-13. Based on this, we deduced that the compound is the deacetylated form of PyA, which we called deacetyl-PyA (DeAc-PyA) (compound 10 shown in Figure 6). However, no DeAc-PyA was found in the control. Neither 11-DeAc-PyO nor DeAc-PyA was detected in the extract of the transformant P450-2 supplied with PyE (data not shown).

\section{DISCUSSION}

Pyripyropenes are meroterpenoids, which are hybrid compounds of both terpenoid and polyketide. PyA has four hydroxyl groups (acetylated at C-1, C-7 and C-11, free C-13-hydroxy). ${ }^{22,23}$ Tomoda et al. have deduced the biosynthetic pathway of PyA from nicotinic acid and mevalonic acid to $(3 S, 6 \mathrm{a} R, 12 \mathrm{~b} S)$-3-hydroxy-4,4,6a,12btetramethyl-9-(pyridin-3-yl)-1,2,3,4,4a,5,6,6a,12,12a-decahydrobenzo [f]pyranol[4,3b]chromen-11(12bH)-one, DeAc-PyE (Figure 6). ${ }^{24-26}$ In this report, nine ORFs ( $p p b 1$ to $p p b 9$ ) for PyA biosynthesis in P. coprobium were deduced. Recently, Itoh et al. ${ }^{21}$ showed the biopathway of PyA in A. fumigatus. However, they elucidated the pathway only for the early steps involving the conversion from nicotinic acid to deacetyl-PyE. The later steps of acetylation and hydroxylation to the final product PyA was unclear. We confirmed the accumulation 

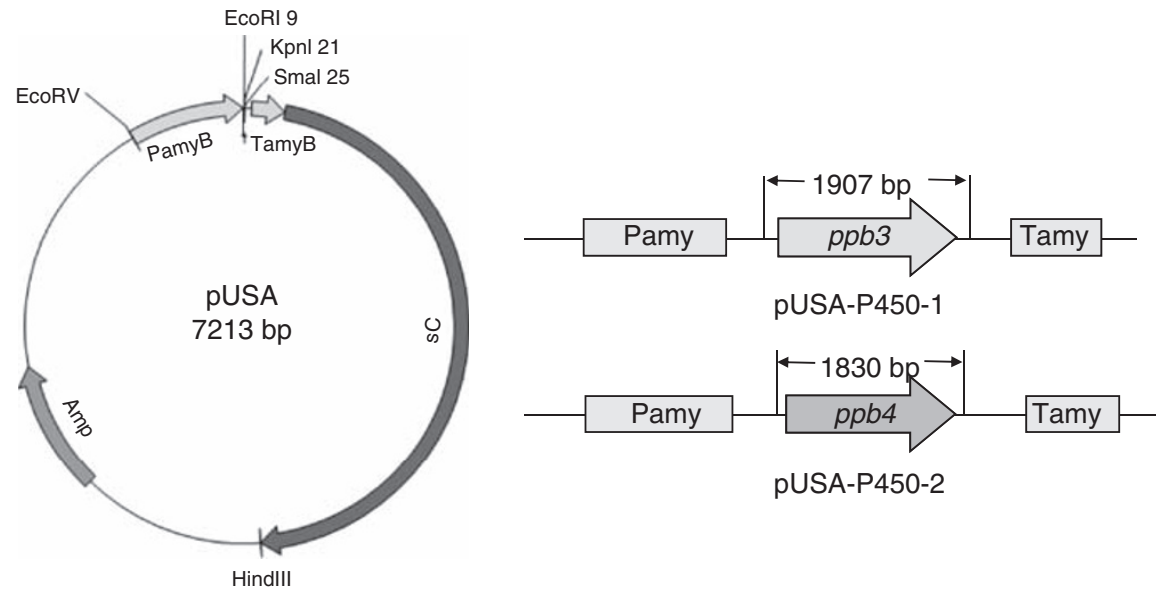

Figure 3 Map of the expression vector and plasmid constructs of pUSA-P450-1 and pUSA-P450-2. PamyB, amylase promoter; TamyB, amylase terminator; sC, ATP-sulfurylase.

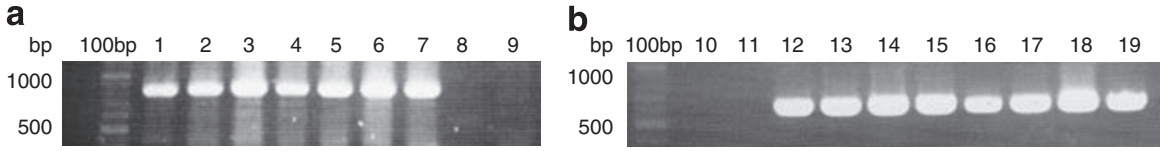

Figure $4 \mathrm{PCR}$ analysis of each transformant to confirm the insert fragment. Prime pairs used are listed in Table 1. (a) With templates of the transformants P450-1, the amplified fragment size is $\sim 830 \mathrm{bp}$. (b) With templates of the transformants P450-2, the amplified fragment size is $\sim 600 \mathrm{bp}$. Lane $1-7$, the transformants that harbor ppb3 gene; Lane 12-19, the transformants that harbor ppb4 gene; Lane 8 and 10, wide type of $A$. oryzae; Lane 9 and 11 , control transformant that harbors an empty vector.

a

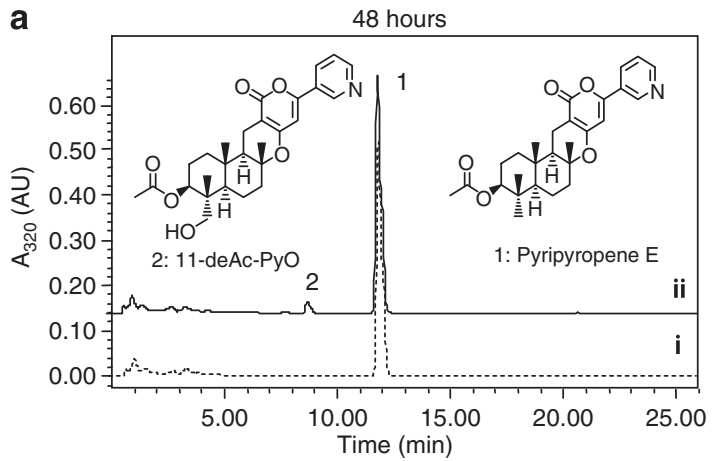

b

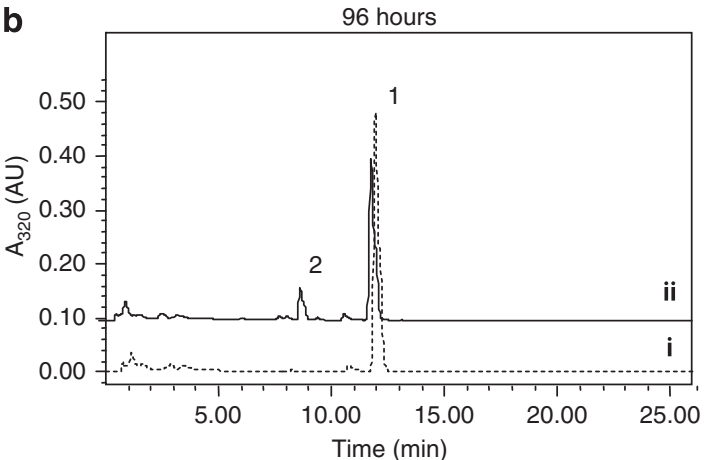

C

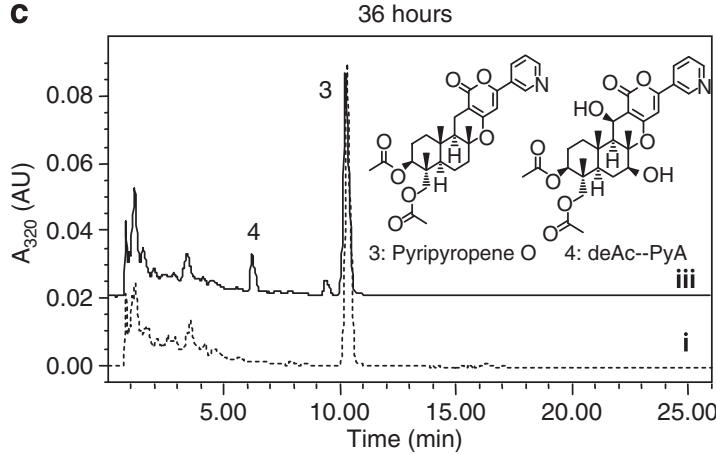

d

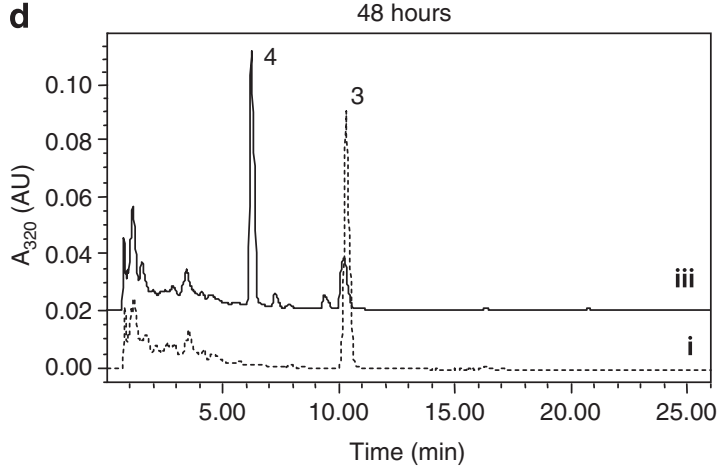

Figure 5 HPLC profile of metabolites. (i) The control transformant that harbors a vector only. (ii) The transformant P450-1 that expresses P450-1. (iii) The transformant P450-2 that expresses P450-2. (a, b) Supplied with the intermediate of PyE. The 11-DeAc-PyO was detected in the extracts of the transformant P450-1. (c, d) Supplied with the intermediate of PyO. DeAc-PyA was detected in the extracts of the transformant P450-2. Flow rates were $0.8 \mathrm{ml} \mathrm{min}^{-1}$ and temperature was $40^{\circ} \mathrm{C}$ in all cases. 

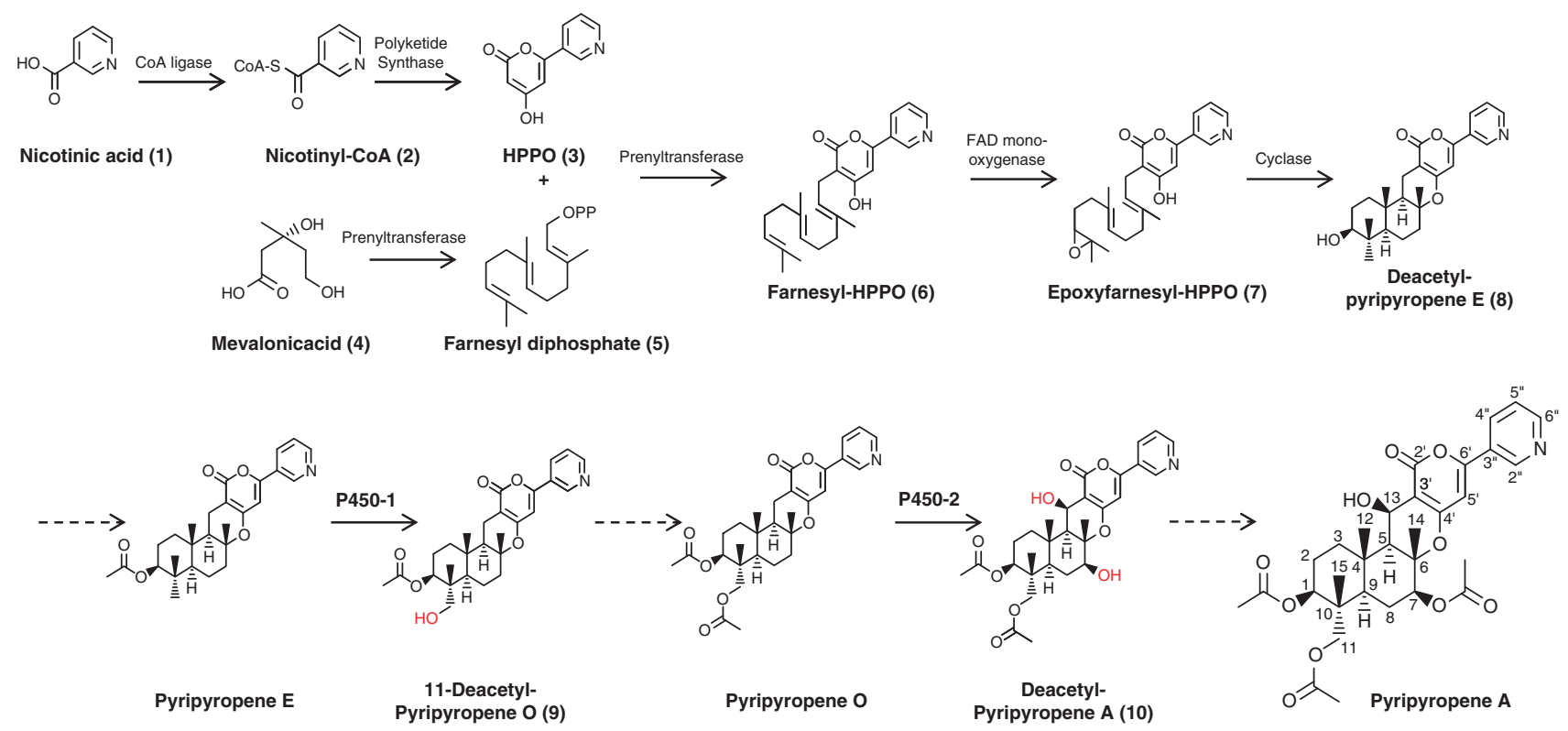

Figure 6 Proposed biosynthetic pathway for Pyripyropene A. Steps from (1) to (8) have been demonstrated by Itoh et al. (2010). Considering the structure of pyripyropene $\mathrm{E}$, pyripyropene $\mathrm{O}$ and pyripyropene $\mathrm{A}$, the next steps were deduced to be acetylation and hydroxylation. This paper shows that P450-1 and P450-2 are involved in hydroxylation at the site of C-11, C-7 and free-C-13.

of PyE and PyO in P. coprobium and deduced that PyE and PyO would be intermediates in the biosynthesis of PyA. Among the nine biosynthetic genes, $p p b 3$ and $p p b 4$ showed high homology to cytochrome P450 monooxygenases. Cytochrome P450 monooxygenases are widely known for their roles in hydroxylation. Consequently, in this study, we focused on these two genes, which are predicted to encode the P450s and presumed to be involved in the hydroxylation of the intermediates in the PyA biosynthetic pathway.

We tried feeding PyE, PyO (Figure 6) and other analogs (data not shown) with transformants harboring the $p p b 3$ or $p p b 4$ gene and detected their bioconversion products. In the extracts of transformants, new compounds, which we would expect to be hydroxylated were found in the transformant $\mathrm{P} 450-1$ with $\mathrm{PyE}$ and in the transformant P450-2 with PyO. On the basis of LC analysis, we detected the bioactivity of the transformant $\mathrm{P} 450-1$ after $48 \mathrm{~h}$ of incubation and the transformant $\mathrm{P} 450-2$ after $36 \mathrm{~h}$ of incubation. The converted ratio increased over time. On the basis of the structure of the PyA, PyE and $\mathrm{PyO}$, and the data from LC-MS and LC-NMR on the extracts of the transformant P450-1 (ppb3) in a feeding test with PyE, we concluded that $\mathrm{C}-11$ of PyE was first hydroxylated by P450-1 monooxygenase and converted to 11-DeAc-PyO. In a feeding test of the transformant P450-2 ( $p p b 4$ ) with PyO, the DeAc-PyA was detected by the same methods. Based on this data and a comparison of the structures of PyO and PyA, we conclude that the $p p b 4$ gene in the transformants P450-2 have active roles in the hydroxylation of C-13 and C-7 of PyO, and conversion to DeAc-PyA. The results also demonstrate that the $p p b 3$ and $p p b 4$ have different roles in this biosynthesis (Figure 6). We were able to elucidate two hydroxylation steps and identified genes involved in the biosynthetic pathway of PyA. However, of all steps in the PyA biosynthesis, only the acetylation steps remain to be confirmed. We were able to deduce the presence of two acetyltransferase genes on the gene cluster. Further functional analysis is currently underway.

PyA is responsible for insecticidal properties, causing a $62 \%$ reduction in weight gain among Helicoverpa zea larvae at dietary levels of 50 p.p.m. (see ref. 2). It shows promise as an insecticide with lower toxicity than conventional insecticides. The isolation and cloning of these enzymes will greatly enhance our understanding of PyA biosynthesis and lead to industrial applications.

\section{ACKNOWLEDGEMENTS}

We wish to express our thanks to Hakutsuru Sake Brewing company for providing the A. oryzae HL-1105 and HL-1034. We also wish to thank Dr Yamada of the National Research Institute of Brewing for providing the plasmid vector pUSA; Dr Yamashita for the documents on transformation; Ms Tsuchida for providing assistance in our LC-MS and LC-NMR analysis; and Dr Pam Kazmierczak and Dr Furutani for reviewing this paper.

1 Tsujiuchi, G., Nakahashi, M., Yamamoto, D., Tani, M. \& Takagi, M. Novel microorganism having ability to produce pyripyropenes. J. Tech. Disclosure. 2008-500997, 2 (2008).

2 Wang, H. J., Gloer, J. B., Wicklow, D. T. \& Dowd, P. F. Aflavinines and other antiinsectan metabolites from the ascostromata of Eupenicillium crustaceum and related species. Appl. Environ. Microbiol. 61, 4429-4435 (1995).

3 Ōmura, S., Tomoda, H., Kim, Y. K. \& Nishida, H. Pyripyropenes, highly potent inhibitiors of Acyl-CoA:Cholesterol acyltransferase produced by Aspergillus fumigatus. J. Antibiot. 46, 1168-1169 (1993).

4 Hayashi, A., Arai, M., Fujita, M. \& Kobayshi, M. Pyripyropenes, fungal sesquiterpenes conjugated with $\alpha$-pyrone and pyridine moieties, exhibits anti-angiogenic activity against human umbilical vein endothelial cells. Biol. Pharm. Bull. 32, 1261-1265 (2009).

5 Tomoda, H. et al. Biosynthesis of pyripyropene A. J. Org. Chem. 61, 882-886 (1996).

6 René De, M. O. T. \& Annabel, H.A.Parret. A novel class of self-sufficient cytochrome P450 monooxygenases in prokaryotes. Trends microbial 10-11, 502-508 (2002).

7 Estabrook, R. W. Cytochrome P450 and Oxygenation Reactions: a Status Report. in Drug Metabolism and Drug Toxicity (eds Mitchell J.R. \& Horning M.G.) 1-20 (Raven Press, New York, 1984).

8 Nelson, D. R. et al. The P450 superfamily: update on new sequences, gen mapping, accession numbers, early trivial names of enzymes, and nomenclature DNA. Cell Biol. 12, 1-51 (1993).

9 Kotze, A. C. Cytochrome P450 monooxygenase activity in Haemonchus contortus (Nematoda). Int. J. Parasitol. 27-1, 33-40 (1997).

10 Yamada, O. et al. dffA gene from Aspergillus oryzae encodes L-ornithine $\mathrm{N}^{5}$-oxygenase and is indispensable for deferriferrichrysin biosynthesis. J. Biosci. Bioeng. 95, 82-88 (2003). 
11 Gomi, K., limura, Y. \& Hara, S. Integrative transformation of Aspergillus oryzae with a plasmid containing the Aspergillus nidulans argB gene. Agric. Biol. Chem. 51, 2549-2555 (1987).

12 Turina, M., Prodi, A. \& Van Alfen, N. K. Role of the Mfl-1 pheromone precursor gene of the filamentous ascomycete Cryphonectria parasitica. Fungal Genet. Biol. 40, 242-251 (2004).

13 Cox, R. J. Polyketides, proteins and genes in fungi: programmed nano-machines begin to reveal their secrets. Org. Biomol. Chem. 5, 2010-2026 (2007).

14 Varga, J., Rigó, K., Kocsube, S., Farkas, B. \& Pál, K. Diversity of polyketide synthase gene sequences in Aspergillus species. Res. Microbiol. 154, 593-600 (2003).

15 Schmitt, I., Kautz, S. \& Lumbsch, H. T. 6-MSAS-like polyketide synthase genes occur in lichenized ascomycetes. Mycol. Res. 112, 289-296 (2008).

16 Nicholson, T. P. et al. Design and utility of oligonucleotide gene probes for fungal polyketide synthases. Chem. Bio. 8, 157-178 (2001).

17 Marco, A.V.S.B., Ilja, W., Chris, L., Richard, K. \& Roel, A. L. B. Functional characterization of the penicillin biosynthetic gene cluster of Penicillium chrysogenum Wisconsin54-1255. Fung. Gene. Biol. 44, 830-844 (2007).

18 Karolewiez, A. \& Geisen, R. Cloning a part of the ochratoxin A biosynthetic gene cluster of Penicillium nordicum and characterization of the ochratoxin polyketide synthase gene. Syst. Appl. Microbiol. 28, 588-595 (2005).

19 Ito, T. et al. O-14, Abstract of paper of 17th Symposium on the Development and Application of Naturally Occurring Drug Materials, Kyushu Univ., Japan (2008).
20 Ito, T. et al. Biosynthetic study of meroterpenoids from filamentous fungi (3)-Functional analysis of enzymes involved in cyclization of terpenoid moiety in the biosynthesis of pyripyropene A. 27G-pm04, Abstract of paper of 129th anniversary conference of the pharmaceutical society of Japan, Kyoto, Japan (2009).

21 Itoh, T. et al. Reconsititution of a fungal meroterpenoid biosynthesis reveals the involvement of a novel family of terpene cyclases. Nat. Chem. 2, 858-864 (2010).

$22 \mathrm{Kim}$, Y. K. et al. Pyripyropenes, novel inhibitors of acyl-CoA:cholesterol acyltransferase produced by Aspergillus fumigatus. II. Structure elucidation of pyripyropenes A, B, C and D. J. Antibiot. 47, 154-162 (1994).

23 Obata, R., Sunazuka, T., Tomada, H., Harigaya, Y. \& Ōmura, S. Chemical modification and structure-activity relationships of pyripyropenes; potent, bioavailable inhibitor of acyl-CoA: cholesterol O-acyltransferase (ACAT). Bioorg. Med. Chem. Lett. 5-22, 2683-2688 (1995).

24 Nakagawa, A. \& Ōmura, S. Biosynthesis of bioactive microbial metabolites and its application to the structural studies and production of hybrid compounds. J. Antibiot. 49-8, 717-741 (1996).

25 Amos, B. S. \& Takeshi, K. Biomimetic total synthesis of the ACAT inhibitor (+)pyripyropene E. Tetrahedron Lett. 37-36, 6461-6464 (1996).

26 Kira, I. \& Onishi, N. Purification and characterization of a (R)-1-phenyl-1,3-propanediol-producing enzyme from Trichosporon fermentns AJ-5152 and enzymatic (R)-1phenyl-1,3-propanediol production. Biosci. Biotechnol. Biochem. 73, 1640-1646 (2009).

Supplementary Information accompanies the paper on The Journal of Antibiotics website (http://www.nature.com/ja) 